\title{
Discharge probability measurement of a Triple GEM detector irradiated with neutrons
}

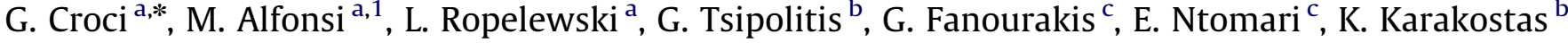 \\ a CERN, Geneva, Switzerland \\ ${ }^{\mathrm{b}}$ National Technical University of Athens, Greece \\ ${ }^{\mathrm{c}}$ Nat. Cent. for Sci. Res. Demokritos, Athens, Greece
}

\section{A R T I C L E I N F O}

\section{Article history:}

Received 15 November 2012

Received in revised form

5 February 2013

Accepted 13 February 2013

Available online 19 February 2013

Keywords:

Triple GEM

Neutrons

Discharge probability

\begin{abstract}
A B S T R A C T
Neutron GEM-based detectors represent a new frontier of diagnostic devices in neutron-linked physics applications such as detectors for fusion experiments (Croci et al., 2012 [1]) and spallation sources (Murtas et al., 2012 [2]). Besides, detectors installed in HEP experiments (like LHC at CERN) are dip in a high flux neutron field. For example, the TOTEM T2 GEM telescope (Bagliesi et al., 2010 [3]) at LHC is currently installed very close to the beam pipe where a high intensity $\left(>10^{4} \mathrm{n} \mathrm{cm}^{-2} \mathrm{~s}^{-1}\right)$ neutron background is present. In order to assess the capability (particularly related to discharge probability) of working in intense neutrons environment, a $10 \times 10 \mathrm{~cm}^{2}$ Triple GEM detector has been tested using a high flux $\left(10^{5} \mathrm{n} \mathrm{cm}^{-2} \mathrm{~s}^{-1}\right)$ neutron beam. The neutron-induced discharge probability $P_{\text {Disch }}$ was measured to be $1.37 \times 10^{-7}$ at an effective gain $G=5 \times 10^{4}$. In addition, the different types of neutron interactions within the detector were fully explained through a GEANT4 simulation.
\end{abstract}

(c) 2013 Elsevier B.V. All rights reserved.

\section{Introduction}

The goal of this test is the study of neutron-induced discharge probability of a Triple GEM detector. The particular detector used in the test has been constructed using the same materials and following the same assembling method as the TOTEM T2 chambers [3].

The TOTEM T2 GEM telescope [3] at LHC is currently installed very close to the beam pipe where a high intensity ( $>10^{4} \mathrm{n} \mathrm{cm}^{-2} \mathrm{~s}^{-1}$ ) neutron background is present. The Triple GEM detectors of the telescope will work at high gains $\left(\geq 10^{4}\right)$ necessary to detect minimum ionizing particles. At such high gains, the very ionizing product generated by neutron reactions can provoke potentially harmful discharges in the detector. In the measurements described in this paper, the discharge probability has been measured for gas gain values from $2 \times 10^{3}$ up to $5 \times 10^{4}$ thus including the gain of T2 GEMs. As a consequence, the detector behavior observed during this irradiation test can be representative of the one of TOTEM T2 GEMs.

More in general, results reported in this paper can be used to foresee the performance, concerning their radiation hardness to neutron induced discharges, of Triple GEM based detectors placed in a high intensity neutron field $[1,2]$.

\footnotetext{
* Corresponding author. Now at: IFP-CNR, Milan, Italy.

E-mail addresses: Gabriele.Croci@cern.ch, croci@ifp.cnr.it (G. Croci).

${ }^{1}$ Now at: NIKHEF, Amsterdam, Nl, The Netherlands.
}

The radiation facility used to perform this test is located in the Demokritos Institute in Athens.

\section{Experimental setup}

\subsection{The Triple GEM detector}

Fig. 1 shows the picture of the detector used to perform the measurements. The GEM foils used in the chamber are standard GEM foils [4].

The detector have a square shaped $\left(10 \times 10 \mathrm{~cm}^{2}\right)$ active area and is provided with a $3 \mathrm{~mm}$ thick drift gap and $2 \mathrm{~mm}$ thick transfers and induction gaps. High voltage is delivered to the chamber through a passive resistive divider (5.4 M $\Omega$ in total) and the potential difference applied to each GEM foil ranges from $300 \mathrm{~V}$ to $400 \mathrm{~V}$. An additional $10 \mathrm{M} \Omega$ resistor is placed in series with each top GEM electrode in order to dump discharges. Although this powering way does not give the possibility to independently change the GEM voltages and the external fields [5], it has been proved to be very robust against discharges. All the read-out board Cartesian $x-y$ strips [6] were shorted together in order to acquire an integrated anode signal.

\subsection{The neutron beam}

Fig. 2 shows a picture of the experimental setup used to irradiate the chamber. 


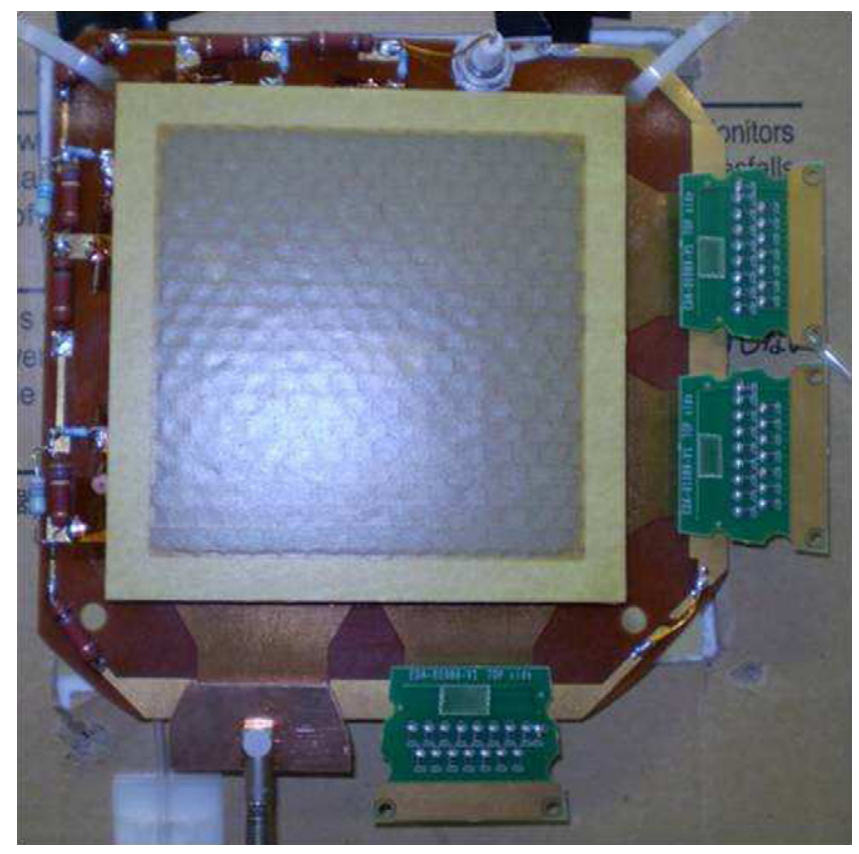

Fig. 1. Photograph of the Triple GEM used in the neutron irradiation test.

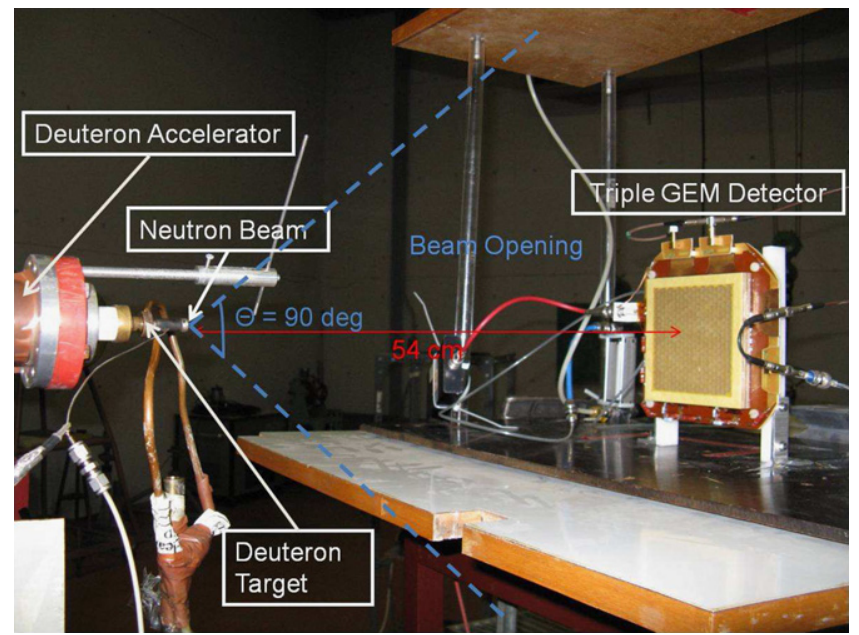

Fig. 2. Experimental setup used to irradiate the Triple GEM detector.

The neutron (5.5 MeV energy) beam is produced by the D-D reaction and has a large opening angle $(\theta=\pi / 2)$, in order to have the possibility to irradiate the full detector active area. Two different rates of neutrons were used during the measurements: $4.4 \times 10^{4} \mathrm{n} \mathrm{cm}^{-2} \mathrm{~s}^{-1}$ and $2.28 \mathrm{n} \mathrm{cm}^{-2} \mathrm{~s}^{-1}$. The employed gas mixture was $\mathrm{Ar} / \mathrm{CO}_{2} 70 \% / 30 \%$.

\subsection{Detector gain measurements}

Fig. 3 shows the measured Triple GEM detector gas gain for different bias voltages applied to the GEM resistor divider. The maximum gas gain used in this test was $5 \times 10^{4}$ corresponding to an applied divider voltage of $4.3 \mathrm{kV}$.

\subsection{Energy Spectrum measurements}

Fig. 4 shows the result of the Energy Spectrum measurement under neutron irradiation (thick light-red curve). The ${ }^{55} \mathrm{Fe}$ Energy Spectrum (thin black line) has been superimposed for calibration purposes.

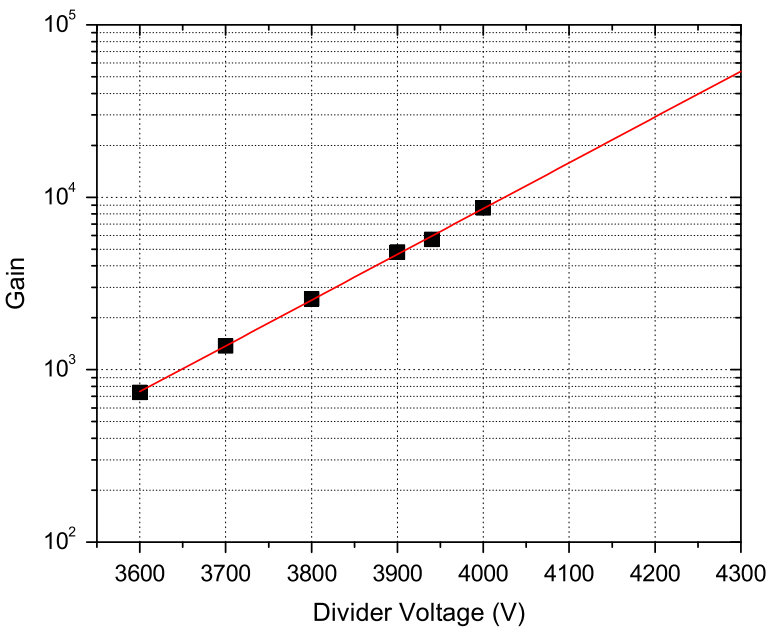

Fig. 3. Triple GEM detector gain measured as function of the divider voltage. During the neutron irradiation test, values of voltage up to $4300 \mathrm{~V}$ were used.

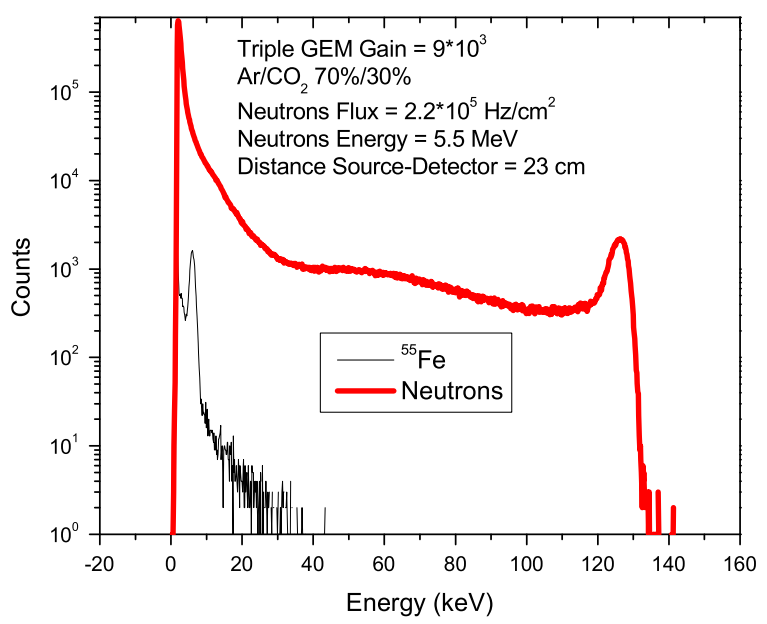

Fig. 4. Energy Spectrum obtained from Triple GEM neutron irradiation (thick light-red curve); the Energy Spectrum of ${ }^{55} \mathrm{Fe}$ is shown for calibration purposes. (For interpretation of the references to color in this figure caption, the reader is referred to the web version of this article.)

In the spectrum, three different components can be distinguished: (i) the slope up to $20 \mathrm{keV}$ is due to interaction of photons (mainly through Compton scattering) coming from the activation of the materials surrounding the detector. Electrons liberated through the Compton scattering process own an energy dependence on the emission angle and can be considered as MIPs: therefore, they contribute to the left part (low deposited energy) of the spectrum. The broad peak (ii) between $40 \mathrm{keV}$ and $90 \mathrm{keV}$ is due to neutrons conversion coming either from the neutron elastic scattering with a gas atom or with the GEM Kapton. Both these interactions generate particles whose energy release is higher than Compton electrons. The peak around $120 \mathrm{keV}$ (iii) is due to the saturation of the employed preamplifier. In this measurement the detector gain was set to 9000 .

The neutrons conversion region in the Energy Spectrum illustrates the response of the detector to neutrons. The Energy Spectrum in Fig. 4 was acquired during $60 \mathrm{~s}$ and the integrated counts below the peak ( $40 \mathrm{keV}<E<100 \mathrm{keV})$ are around 435,550 , leading to a measured neutron interaction rate $R_{\text {Meas }}=7260 \mathrm{~Hz}$. 
If pure Argon is considered to be the gas mixture, the expected neutron interaction rate with the gas, $R_{\text {exp }}$, can be estimated as

$R_{\text {exp }}=F \cdot N_{A r} \cdot \sigma$

where $F$ is the neutron flux, $N_{A r}$ is the number of Argon atoms contained in the drift volume and $\sigma$ is the sum of the crosssections for elastic and inelastic scattering between neutrons and the Ar atoms. Since $F=2.28 \times 10^{5} \mathrm{n} \mathrm{cm}^{-2} \mathrm{~s}^{-1}, N_{A r}=8.428 \times 10^{21}$ and $\sigma=4 \mathrm{~b}, R_{\text {exp }}=7680 \mathrm{~Hz}$. $R_{\text {exp }}$ and $R_{\text {Meas }}$ have compatible values.

\section{GEANT4 simulation}

A simulation using the GEANT4 [7] tool has been performed in order to discriminate the contributions to the deposited energy due to different neutrons interactions.

\subsection{The simulated setup}

The geometry of the setup in the simulation was simplified with respect to the real setup and resembles a single-GEM like detector.

Fig. 5 shows a picture of the simulated setup: the beam direction defines the $z$-axis, so that neutrons enter the detector perpendicular to the drift electrode. The neutron source is monochromatic $\left(E_{n}=5.5 \mathrm{MeV}\right)$, located $20 \mathrm{~cm}$ far away from the GEM center and Gaussian distributed in $x$ and $y$ (with $\sigma_{x}=\sigma_{y}=$ $0.2 \mathrm{~mm}$ ).

Table 1 describes the components of the detector that were included in the simulation as well as their material. The $x$ and $y$ dimensions of all the elements are $10 \mathrm{~cm} \times 10 \mathrm{~cm}$.

The gas mixture used in the simulation is $\mathrm{Ar} / \mathrm{CO}_{2} 70 \% / 30 \%$.

All materials have been created by retrieving each composing element from the GEANT4 NIST database. The physics processes are defined by the GEANT4 physics list especially suited for neutrons (QGSP_BERT_HP).

The active part of the detector is the gas volume: only the energy released in the gas by the particles created by primary neutrons is recorded. Since the aim of this simulation is to discriminate the contribution of the different neutrons interaction processes to the energy release in the gas, no electric field has been applied between the different electrodes, no holes have been created in the GEM foils and drift of electrons and ions pairs liberated into the gas is not simulated. The energy released in the gas by a particle generated by a neutron interaction constitutes one entry in the histogram of Fig. 6.

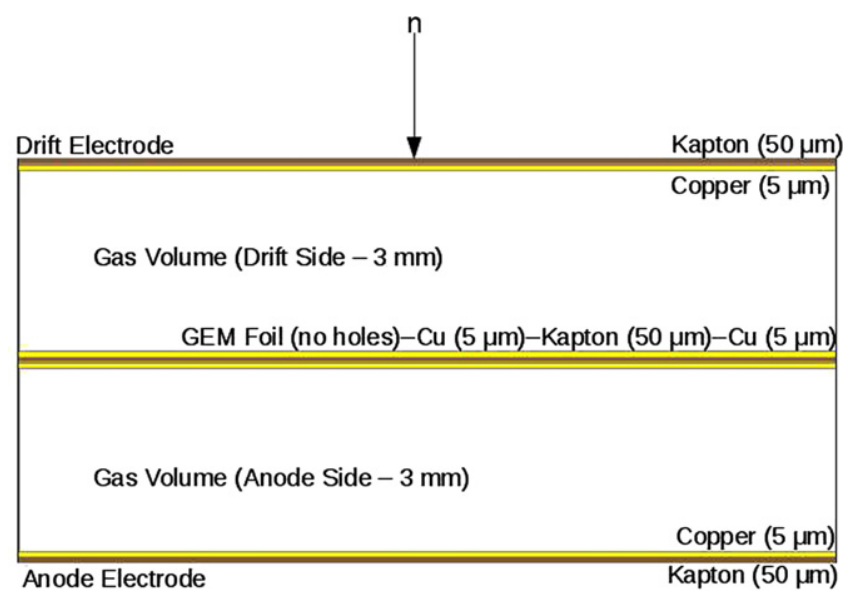

Fig. 5. The setup simulated using Geant4. The neutron beam enters the detector perpendicularly to the drift electrode.
Table 1

Geometrical parameters and materials of the detector components included in the simulation.

\begin{tabular}{llll}
\hline Component & Layer & $z$-Dim & Material \\
\hline Drift electrode & Kapton & $50 \mu \mathrm{m}$ & Kapton \\
Drift electrode & Copper & $5 \mu \mathrm{m}$ & Copper \\
Gas volume (drift side) & Gas & $3 \mathrm{~mm}$ & $\mathrm{Ar} / \mathrm{CO}_{2}$ \\
GEM foil & Top electrode & $5 \mu \mathrm{m}$ & $\mathrm{Copper}$ \\
GEM foil & Kapton & $50 \mu \mathrm{m}$ & $\mathrm{Kapton}$ \\
GEM foil & Bottom electrode & $5 \mu \mathrm{m}$ & $\mathrm{Copper}$ \\
Gas volume (anode side) & Gas & $3 \mathrm{~mm}$ & $\mathrm{Ar} / \mathrm{CO}_{2}$ \\
Anode electrode & Copper & $5 \mu \mathrm{m}$ & $\mathrm{Copper}$ \\
Anode electrode & Kapton & $50 \mu \mathrm{m}$ & $\mathrm{Kapton}$ \\
\hline
\end{tabular}

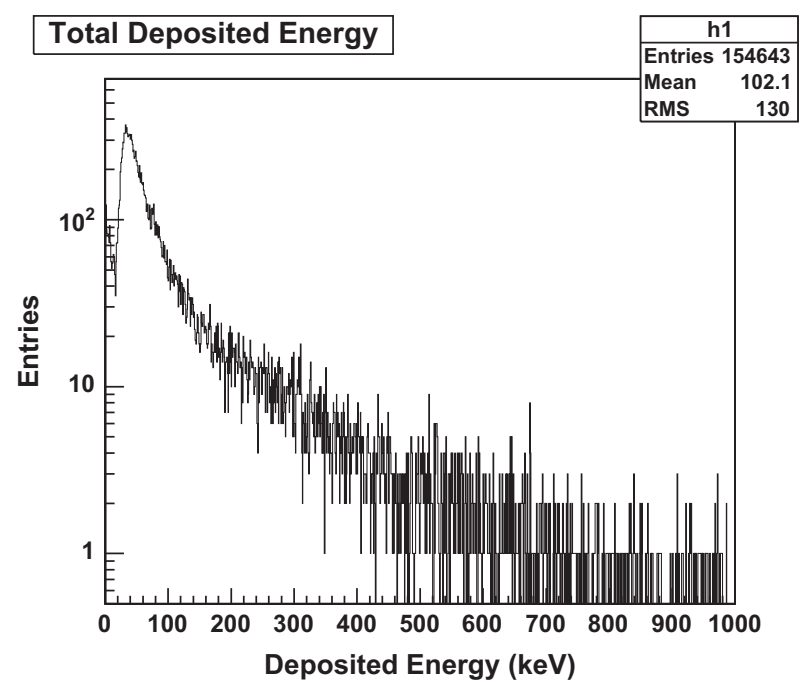

Fig. 6. Histogram of the total deposited energy in the gas volume due to neutron interactions.

\subsection{Simulations results}

A simulation of about $10^{8}$ primary neutrons has been performed in order to get a statistically significant result.

Fig. 6 shows the deposited energy in the gas due to neutrons interactions.

A peak at about $60-70 \mathrm{keV}$ corresponding to the same peak energy of the experimental Energy Spectrum shown in Fig. 4 is present.

A list of some of the recognized secondary particles created through the interaction between neutrons and detector materials is reported in Table 2 .

Among all the interactions, the most probable ones are the elastic scattering between neutron and Argon Ion $\left({ }^{40} \mathrm{Ar}\right)$, Carbon Ion $\left({ }^{12} \mathrm{C}\right)$, Oxygen Ion $\left({ }^{8} \mathrm{O}\right)$ and hydrogen in the plastic materials. Interactions with hydrogen generate protons through the neutron-proton elastic scattering process.

Fig. 7 shows that almost all the deposited energy comes from two of these four main processes. The process of neutron elastic scattering on Argon atoms is responsible for the tail at low energy (up to $30 \mathrm{keV}$ ), while the peak comes from the energy released by protons in the gas.

Fig. 8 illustrates the distribution of initial positions of secondary particles showing the role of the different materials to their generation.

Solid materials (electrodes and Kapton foil) as well as the gas, are both sources of secondary particles. Particles created in the GEM foil by neutron beam interactions are mainly emitted in the same direction of the beam because of momentum conservation: 
Table 2

Some of the secondary particles created by primary neutrons.

\begin{tabular}{l}
\hline Secondary particles \\
\hline${ }^{40} \mathrm{Ar}$ \\
$\gamma$ \\
$\mathrm{n}+\gamma$ \\
$\mathrm{p}$ \\
${ }^{12} \mathrm{C}$ \\
$\mathrm{n}+{ }^{40} \mathrm{Ar}$ \\
${ }^{16} \mathrm{O}$ \\
$\mathrm{n}+{ }^{12} \mathrm{C}$ \\
$\mathrm{n}+{ }^{63} \mathrm{Cu}+\gamma$ \\
$\mathrm{p}+\gamma$ \\
${ }^{4} \mathrm{He}+\gamma$ \\
$\mathrm{p}+{ }^{63} \mathrm{Ni}$ \\
$\mathrm{n}+{ }^{36} \mathrm{Ar}$ \\
${ }^{63} \mathrm{Cu}$ \\
${ }^{65} \mathrm{Cu}$ \\
\hline
\end{tabular}

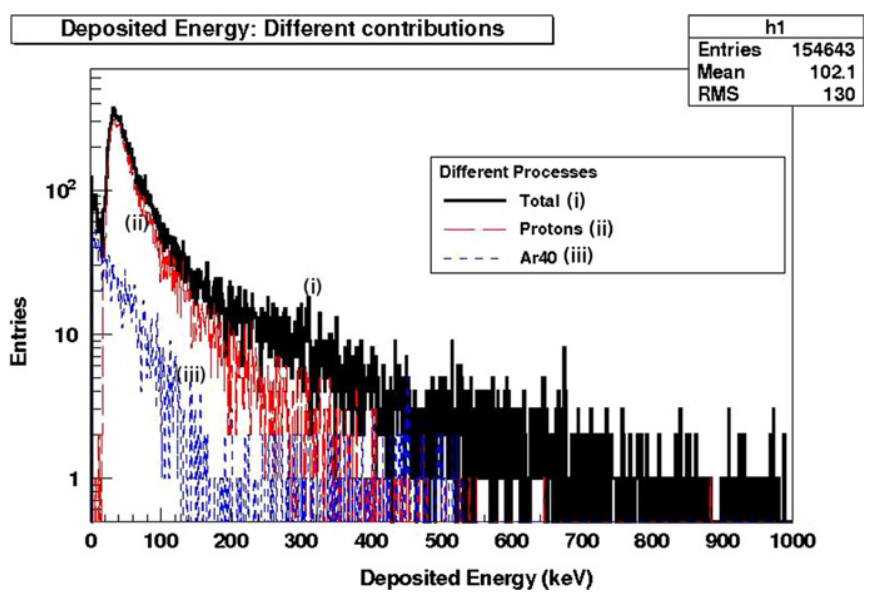

Fig. 7. Different contributions to total energy deposition in the gas volume. Neutron interactions with $\mathrm{H}$ (ii, long dashed line) and $\mathrm{Ar}$ (iii, short dashed line) atoms are responsible for almost all the deposited energy in the detector. The process of neutron elastic scattering on Argon atoms generates the tail at low energy (up to $30 \mathrm{keV}$ ), while the peak at $60-70 \mathrm{keV}$ comes from interactions between protons and gas.

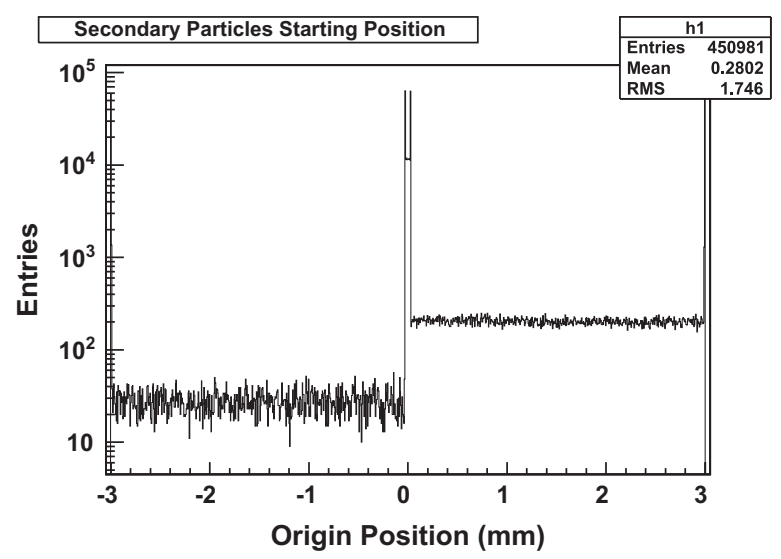

Fig. 8. Histogram of starting position of the secondary particles generated in the detector by primary neutrons interactions.

as a consequence a higher number of particles is generated by the products of the neutron reactions in the induction volume.

Figs. 9 and 10 illustrate that the protons are produced by the interaction between neutrons and solid materials, in particular between neutrons and Kapton foils.

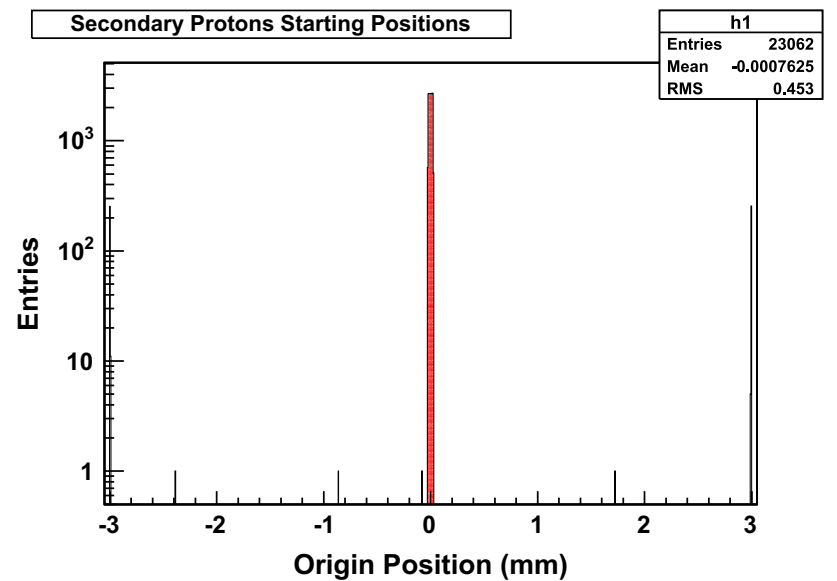

Fig. 9. Histogram of starting position of secondary protons: the main contribution comes from conversions in solid materials.

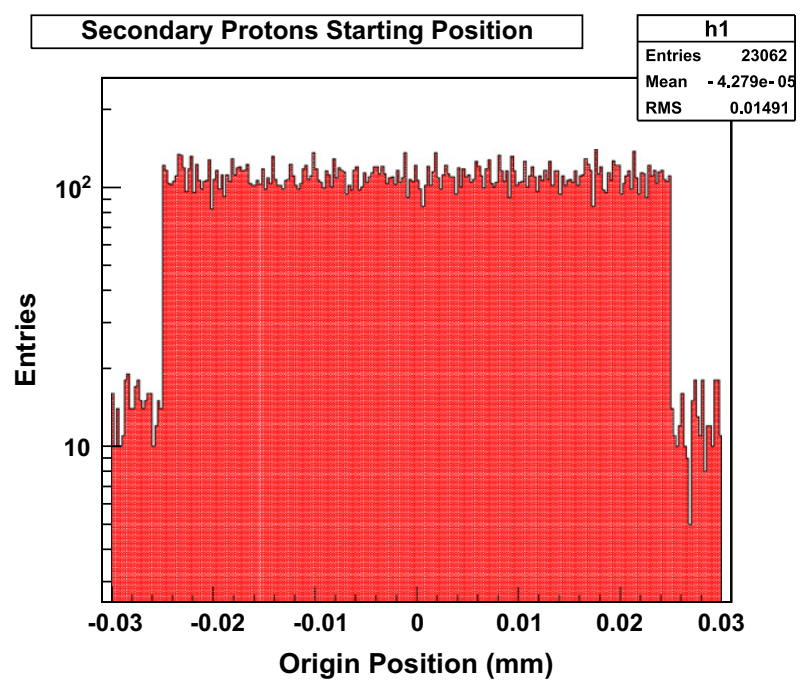

Fig. 10. Histogram of starting position of secondary protons: zoom in the region of the GEM Kapton foil. As expected, the main contribution comes from neutron conversion in this material.

Since one element composing the Kapton material (chemical formula $\mathrm{C}_{22} \mathrm{H}_{10} \mathrm{~N}_{2} \mathrm{O}_{5}$ ) is hydrogen, elastic scattering of a neutron with hydrogen would produce a proton with a cross-section of about $1.5 \mathrm{~b}$ at $E_{n}=5.5 \mathrm{MeV}$. These protons have enough energy to cross the electrodes, reach the gas and ionize it.

\section{Neutrons irradiation: measurement of the discharge probability}

\subsection{Measurements description}

In order to measure the Triple GEM discharge probability under neutron irradiation, a current measurement has been performed. The current was read out only from the anodic strips (connected all together) using a picoamperometer with $\sim 1 \mathrm{pA}$ resolution. Since the current was measured on the anode, and not on all GEM electrodes, only the consequence of a discharge could be observed. If a discharge occurs in one of the three GEMs, the corresponding potential difference will drop to zero and will come back to its set value during a period that is defined by the RC time of the GEM foil. As a consequence, the gain of one of the three GEMs is temporarily reduced and a lower current (in 
Table 3

Experimentally defined current variation $\left(\Delta I_{\text {Disch }}\right)$ used to identify discharges for different gains.

\begin{tabular}{rc}
\hline \multicolumn{1}{l}{ Gain } & $\Delta I_{\text {Disch }}(\mathrm{nA})$ \\
\hline $2.8 \times 10^{3}$ & 2.8 \\
$5 \times 10^{3}$ & 5 \\
$9 \times 10^{3}$ & 9 \\
$1.8 \times 10^{4}$ & 18 \\
$3 \times 10^{4}$ & 30 \\
$5 \times 10^{4}$ & 50 \\
\hline
\end{tabular}

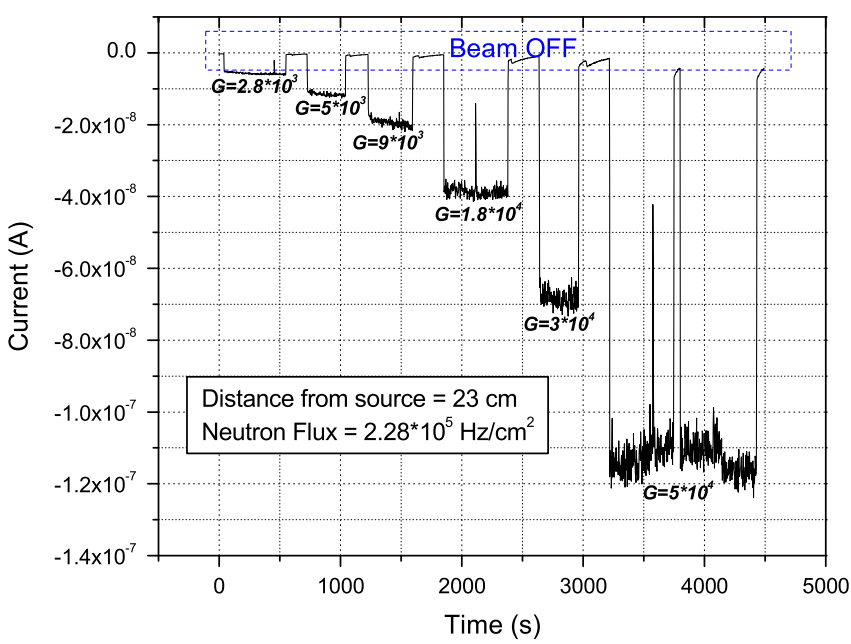

Fig. 11. Real time measurement of discharge probability. The anodic current has been measured for five different gain $(G)$ values for at least $700 \mathrm{~s}$ with a neutron flux $=2.28 \times 10^{5} \mathrm{n} \mathrm{cm}^{-2} \mathrm{~s}^{-1}$. Three current drops have been observed, respectively, for $G=2.8 \times 10^{3}, G=1.8 \times 10^{4}$ and $G=5 \times 10^{4}$.

absolute value) is measured on the anode. In this experiment, a discharge has been defined as a current drop higher than an experimentally defined current variation $\left(\Delta I_{\text {Disch }}\right)$ that has a different value for each detector gain (see Table 3 ).

\subsubsection{Results}

Fig. 11 illustrates the real time measurement of discharge probability.

During the measurement, the voltage on the divider was modified in order study the response of the detector at different gains. The period when the current value is zero corresponds to the time during which the neutron beam was off. A constant (negative) current has been measured during neutron irradiation for different gains. Three current drops have been measured during the measurement period (around $1 \mathrm{~h}$ and $15 \mathrm{~m}$ ): these drops can be due either to the effect of discharges or to the temporary lack of the beam. Considering them as discharges (since all of them exceeded the experimentally defined current variation needed to identify a discharge), the upper limit of the neutron induced discharge probability $P_{\text {Disch }}$ can be calculated as follows:

$P_{\text {Disch }}=\frac{N_{\text {Disch }}}{R_{\text {meas }} \cdot \Delta T_{\text {meas }}}$

where $R_{\text {meas }}$ is the measured neutron interaction rate and $\Delta T_{\text {meas }}$ is the measurement period.

In the case of the maximum gain used in the measurement, since $R_{\text {meas }}=7260 \mathrm{~Hz}$ (see Section 2.4 ), $N_{\text {Disch }}=1$ and $\Delta T_{\text {meas }}=$ $1000 \mathrm{~s}, P_{\text {Disch }}=1.37 \times 10^{-7}$ at $G=5 \times 10^{4}$.

This result shows that in GEM-based detectors neutrons induced discharge probability is lower than alphas induced discharge probability [4].

\section{Conclusions}

The neutrons induced discharge probability of Triple GEM detector has been proved to be negligible $\left(P_{\text {Disch }}=1.34 \times 10^{-7}\right.$ at $G=5 \times 10^{4}$ ) even for gas gain as high as $5 \times 10^{4}$; no decrease of performance have been observed in this detector.

The Energy Spectrum obtained under neutron irradiation has been explained by the GEANT4 simulation. In addition, the simulation shows that in GEM-based detector the Kapton foil is the most important source of secondary particles, mainly protons. Therefore, it is possible to conclude that GEM based detectors can be safely and efficiently used in a intense neutron flux environment and that the discharge probability due to neutrons is lower than the discharge probability due to alpha particles.

\section{Acknowledgments}

This work was performed in the framework of the RD51 collaboration.

\section{References}

[1] G. Croci, et al., Journal of Instrumentation 7 (2012) C03010.

[2] F. Murtas, et al., Journal of Instrumentation 7 (2012) P07021.

[3] M.G. Bagliesi, et al., Nuclear Instruments and Methods in Physics Research Section A 617 (2010) 134.

[4] S. Bachmann, et al., Nuclear Instruments and Methods in Physics Research Section A 479 (2002) 294.

[5] C. Altunbas, et al., Nuclear Instruments and Methods in Physics Research Section A 490 (2002) 177.

[6] A. Bressan, et al., Nuclear Instruments and Methods in Physics Research Section A 425 (1999) 254.

[7] S. Agostinelli, et al., The GEANT4 Collaboration, Nuclear Instruments and Methods in Physics Research Section A 506 (2003) 250. 